\title{
Mobile Accessibility Workshop
}

\author{
Daniel Gonçalves ${ }^{1}$, Luis Carriço ${ }^{2}$, and Markel Vigo ${ }^{3}$ \\ ${ }^{1}$ Instituto Superior Técnico / TULisbon, \\ Rua Alves Redol 9, 1000-029 Lisboa, Portugal \\ daniel.goncalves@inesc-id.pt \\ ${ }^{2}$ Faculdade de Ciências / University of Lisbon, \\ Edifício C6, Piso 3. Campo Grande, 1749-016 Lisboa, Portugal \\ lmc@i.fc.ul.pt \\ ${ }^{3}$ School of Computer Science / University of Manchester, \\ LF1 Kilburn Building, Oxford Road, M13 9PL, Manchester, UK \\ markel.vigo@manchester.ac.uk
}

\begin{abstract}
In this document we propose the creation of a Mobile Accessibility Workshop at Interact 2011. Mobile Accessibility is an area that has grown both in importance and number of researchers in recent years. Bringing them together in a workshop would be fruitful and lead to synergies and major developments in the area.
\end{abstract}

Keywords: Mobile Accessibility, mobile computing, accessibility, workshop.

\section{Topic and Objectives}

In recent years, we have witnessed an increasing importance of mobile devices. They pervade our daily lives, not only just in the form of feature phones, but also as smartphones and tablets. Tablets in particular have been the object of much attention, lately. They are set to be one of the fastest growing mobile device markets. What is more, they have the computing power of small computers. This is also true for the most advanced smartphones and mobile operating systems. We are, thus, in the cusp of a fundamental change in how we relate to information and others, accessible at all times and places with the help of mobile devices.

Alas, this change might be barred to a large number of people, suffering from a wide range of disabilities. Mobile devices are increasingly visual, making them hard to use by the blind or other vision-impaired people. Keyboards are steadily being replaced by touchscreens, without tactile feedback, introducing additional barriers. Tetraplegic users have limited mobility of their limbs and hardly are able to pick up the devices or use their fingers for precise pointing. Additionally, mobile devices are being used as mediators to reach distal interfaces (e.g. interactive TV, information kiosks, etc.) providing people with disabilities with the means to potentially overcome physical barriers and freely interact with the environment.

Moreover, the mobile aspect often creates situations where those interaction modes are cumbersome or socially unacceptable, even for non-impaired people. Riding a bicycle on a mountain trail and looking at the maps at the same time is difficult and dangerous. Timely responding to urgent and confidential solicitations on a public site 
may be distressing or embarrassing. These are considered situational disabilities that often introduce similar problems to the use of mobile apparatus. Overall, the list of problems is immense.

Fortunately, there is a nascent area of research, Mobile Accessibility, where researchers are focusing on solving accessibility problems in mobile devices and settings. Bringing together Mobile Computing and Accessibility, it is the source of synergistic works that have the potential of deeply transforming how we look at mobile devices, and to shape the ongoing mobile revolution. Work in this area ranges from new text introduction techniques to multimodal interaction solutions, mobile assistive technology, prototyping tools, navigation tools, theoretical models, etc. A wide range of users and situations is also considered (blind, tetraplegic, elderly, deaf, etc.). As a result, solutions provided to people with disabilities could be applied to the aforementioned situationally impaired users.

Papers on Mobile Accessibility have become an increasing presence in conferences such as CHI, Assets, W4A and MobileHCI, proof of a growing and active community. However, there has been a lack of a single well defined venue for the topmost researchers in the area to meet and exchange ideas and results. We propose, in this first Mobile Accessibility Workshop, to provide such a venue. It is our objective to bring together researcher and practitioners with clear advantages in terms of critical mass, synergies, collaborations and networking, and unmistakable benefits for the area.

\section{Participants}

Organizers: The workshop will be organized by Prof. Daniel Gonçalves, Prof. Luís Carriço and Markel Vigo, all of them researchers in mobile accessibility, with several papers published in the area.

Intended Audience: Researchers and practitioners of Mobile Accessibility, both from academia and industry, in any of its facets (as described in the introduction).

Expected participants: we expect to attract 12-18 participants.

\section{Organization}

Duration: the workshop will last an entire day

Format: the workshop will consist, mainly, of peer-reviewed paper presentations by its participants. We'll solicit submissions using the Interact short paper template.

Extended versions of the best papers will be chosen for submission to a Special Issue of the Universal Access in the Information Society Journal 